\title{
Estimating the radiation dose to the fetus during prophylactic internal iliac occlusion in patients with abnormal placentation
}

\author{
Marie Cerna ${ }^{\mathrm{a}}$, Karel Huml ${ }^{\mathrm{b}}$, Martin Kochera, Lenka Jonasova ${ }^{\mathrm{c}}$, Tomas Vavra ${ }^{\mathrm{a}}$, Vojtech Prasil ${ }^{\mathrm{a}}$
}

\begin{abstract}
Aims. To evaluate the estimated fetal radiation dose during prophylactic internal iliac arterial occlusion in patients with abnormal placenta and to estimate the risk of radiation induced cancer in child age.

Methods. Prophylactic occlusion of the internal iliac arteries during Caesarean section was performed in 42 patients with placenta praevia and/or placenta accreta spectrum. Fogarty embolectomy catheters were used for prophylactic occlusion of the internal iliac arteries. All procedures were performed in the hybrid operating room using Philips Allura Xper FD 20 X-ray system. Low dose X-ray fluoroscopy (7.5 frames per second) was used. The CODE (Conceptus dose estimation) Software was used to estimate the fetal dose and the risk of radiation induced carcinoma.

Results. Fluoroscopy times required for insertion of Fogarty catheters were 0.5-4.2 min (mean: 1.7 min, median: 1.5 $\mathrm{min}$ ). The estimated radiation dose to the fetus was 0.26-3.36 mGy (mean: $1.49 \mathrm{mGy}$, median: $1.25 \mathrm{mGy}$ ). The risk of radiation induced cancer in child age was $0.01-0.04 \%$ (mean $0.02 \%$, median $0.01 \%$ ). One patient developed thrombosis of a common femoral artery.

Conclusion. Prophylactic occlusion of the internal iliac arteries is a simple and safe procedure with minimal risk of complications and with a very low estimated radiation dose to the fetus.
\end{abstract}

Key words: placenta praevia, placenta accreta spectrum, prophylactic occlusion, internal iliac arteries, fetus radiation dose

Received: May 18, 2020; Revised: September 15, 2020; Accepted: October 2, 2020; Available online: November 26, 2020 https://doi.org/10.5507/bp.2020.046

(c) 2021 The Authors; https://creativecommons.org/licenses/by/4.0/

${ }^{a}$ Department of Radiology, University Hospital Olomouc and Faculty of Medicine and Dentistry Palacky University Olomouc, Czech Republic ${ }^{b}$ Department of Obstetrics and Gynaecology, University Hospital Olomouc and Faculty of Medicine and Dentistry, Palacky University Olomouc, Czech Republic and Department of Obstetrics and Gynaecology, Bata Hospital Zlin, Czech Republic

'Department of Medical Physics and Radiation Protection, University Hospital Olomouc, Czech Republic

Corresponding author: Marie Cerna, e-mail: mcernam@fnol.cz

\section{INTRODUCTION}

Placenta praevia (complete or marginal) is defined as abnormal placentation near or covering the internal cervical os. Placenta accreta spectrum, previously known as morbidly adherent placenta, is defined as an abnormal trophoblast invasion of all or part of the placenta to the uterine wall. Placenta accreta spectrum includes placenta accreta, placenta increta, and placenta percreta.

Placenta praevia and accreta spectrum are associated with a high risk of maternal and fetal mortality and morbidity, especially when diagnosed during delivery. These placental disorders, in particular abnormal placenta invasion, are associated with a risk of massive bleeding ${ }^{1,2}$. Prenatal diagnosis by ultrasonography and/or magnetic resonance imaging is crucial to reduce maternal blood loss and postpartum complications. Delivery by Caesarean section can be planned and performed in a specialized centre, where a multidisciplinary team is available ${ }^{3,4}$.

Recently, efforts have been made in patients with placental disorders to avoid hysterectomy and preserve fertility ${ }^{5}$. Temporary prophylactic bilateral internal iliac artery occlusion may allow Caesarean delivery to be performed with a reduced risk of intraoperative bleeding and without hysterectomy. Prophylactic occlusion of the internal iliac arteries is performed under fluoroscopic guidance with potential risks related to ionizing radiation. Nevertheless, the risks of fetal effects, including inducing childhood cancer, are thought to be negligible at fetal doses $<50$ mGy $\left(\right.$ ref. $\left.^{6}\right)$.

The aim of our retrospective study, from prospectively collected data, was to evaluate the estimated fetal radiation dose associated with prophylactic internal iliac occlusion during Caesarean section in patients with abnormal placenta (placenta praevia and placenta accreta spectrum) and to estimate the risk of radiation induced cancer in child age.

\section{MATERIAL AND METHODS}

From November 2015 to November 2019, we performed prophylactic occlusion of the internal iliac arteries in 42 pregnant patients of mean age 35 years (range 23-48 years). The combined procedure of prophylactic internal iliac artery occlusion and Caesarean section was indicated in pregnant patients with abnormal placentation. Prophylactic occlusion of the internal iliac arteries during Caesarean section was performed to decrease blood loss and avoid hysterectomy. The indication for prophylactic 
occlusion of the internal iliac arteries in patients with placenta percreta was a reduction in blood loss during hysterectomy. At our hospital hysterectomy is primarily indicated in placenta percreta and attempts to remove placenta percreta are not performed. All consecutive patients with placenta previa or accreta spectrum were included in this study.

Placental abnormalities were diagnosed prenatally, in all 42 patients by ultrasonography. In 31 patients magnetic resonance imaging was also performed. Placenta previa was diagnosed in 21 patients, placenta praevia and adherens in 3, placenta praevia and placenta accreta in 13 patients, and placenta praevia and percreta in 5 patients.

\section{Endovascular technique}

All procedures were performed in a hybrid operating room with a Philips Allura Xper FD 20 X-ray system.

The cross-over technique was used for placement of introducers and occlusion balloons. Introducer sheaths used were $55 \mathrm{~cm}$ hydrophilic, shaped $6 \mathrm{~F}$ introducers (Flexor Touhy-Borst Side-Arm Introducer, Ansel modification 2 - Renal Double, Cook, Bloomington, IN). The shape of these introducers allows easy cross-over catheterisation, almost always without requiring preshaped catheter. The correct position of introducers was verified by injection of contrast medium. For temporary occlusion of the internal iliac arteries, 5.5F over-the-wire Fogarty thru-lumen embolectomy catheters were used (Edwards Lifesciences, Irvine, CA, USA). An advantage of these catheters is the ability to occlude arteries of different diameters and the possibility to flush the internal iliac arteries and catheters through the lumen of the embolectomy catheter. Successful occlusion of the internal iliac arteries by the inflated Fogarty balloons was tested with contrast medium and the volume of contrast medium in the balloons was recorded. Introducers were continuously flushed with saline by pressure infusion to minimize the risk of thrombosis. Introducers and Fogarty balloons were firmly fixed to the surgical drape to prevent migration. Thereafter, Caesarean section was performed under general anaesthesia. After delivery of the neonate and umbilical cord clamping, the balloons were blindly inflated to reduce bleeding in the operating field, and delivery of the placenta and uterine suture were completed or hysterectomy in case of placenta percreta was done. After surgery, the femoral arteries were percutaneously closed using a nitinol clip (StarClose SE, Abbott Vacular, Santa Clara, CA, USA).

We used low dose X-ray fluoroscopy (7.5 frames per second) for Fogarty embolectomy catheter insertion to minimise radiation dose (Fig. 1). Digital subtraction angiography record was not performed in any patients.

This study used prospectively collected data from all patients for calculation of the fetal dose. The data were recorded during every procedure. The data needed to estimate the fetal dose are stage of pregnancy, fluoroscopy time, X-ray tube voltage, study area including projection, zoom, total DAP (dose area product), distance FSD (focus-to-skin distance), filtration $(\mathrm{Al} / \mathrm{Cu})$ (Table 1). The CODE (Conceptus dose estimation) Software was used to estimate the fetal dose and the risk of radiation induced cancer in child age. The software was developed for the calculation of fetal dose for different stage of pregnancy.

\section{RESULTS}

The technical success rate of Fogarty catheter insertion was $100 \%$. Fluoroscopy times required for the insertion of Fogarty catheters were 0.5-4.2 min (mean: $1.7 \mathrm{~min}$, median: $1.5 \mathrm{~min}$ ) (Table 1). The mean estimated radiation dose to the fetus was $1.49 \mathrm{mGy}$, the median was $1.25 \mathrm{mGy}$ (0.26-3.36 mGy) (Table 1).

The risk of radiation induced cancer in child age was 0.01-0.04\% (average risk: $0.02 \%$, median risk: $0.01 \%$ ) (Table 1). One patient developed thrombosis of a common femoral artery and this complication was successfully managed by surgical thrombectomy.

Hysterectomy was performed in 6 patients, five patients with placenta percreta and one patient with placenta accreta. The indication for hysterectomy in the patient with placenta accreta was massive bleeding during an attempt to remove of the placenta. We did not observe dislocation of the occlusion balloons during the hysterectomy.

Embolisation of the uterine arteries with spongostan (Gelita-Spon standard, Gelita medical, Eberbach, Germany) was successfully performed in 7 patients during the procedure because of postoperative bleeding.

\section{DISCUSSION}

Prophylactic occlusion of the internal iliac arteries is indicated in pregnant women with placental abnormalities (placenta praevia and accreta spectrum) to reduce blood loss during Caesarean section and to avoid hysterectomy and preserve fertility ${ }^{7-11}$.

Prophylactic temporary occlusion of the internal iliac arteries is a fluoroscopically guided procedure. The use of fluoroscopy is associated with potential adverse effects to the fetus due to the use of ionizing radiation. The probability of inducing cancer increases with the radiation dose. Estimated radiation doses to the fetus during prophylactic occlusion of the internal iliac arteries are low $^{12,13}$. Some risk of radiation inducted cancer exists and the risk is not zero at doses of the order of $10 \mathrm{mGy}$ (ref. ${ }^{14}$ ). The risk of radiation induced fatal cancer in childhood is about $0.06 \%$ per $10 \mathrm{mGy}(1 / 1700)\left(\right.$ ref. $\left.^{15}\right)$. According to practice guidelines of ACOG (The American College of Obstetricians and Gynecologists), the risk of fetal effects, including childhood cancer induction, are thought to be negligible at fetal doses $<50 \mathrm{mGy}\left(\right.$ ref. $^{6}$ ). According to ICRP (The International Commission on Radiological Protection), a fetal dose of $100 \mathrm{mGy}$ has a small individual risk of radiation-induced cancer. There is over a $99 \%$ chance that the exposed fetus will not develop childhood cancer ${ }^{16}$.

Various aspects of the procedure, namely the medical devices used, the mode of fluoroscopy, and use of collimation contribute to a reduce fluoroscopy time and thus the fetal dose. The use of hydrophilic coated preformed sheaths allows easy crossing of the aortic bifurcation di- 
Table 1. The parameters and results of patients.

\begin{tabular}{|c|c|c|c|c|c|c|c|c|}
\hline Patient & $\begin{array}{c}\text { Voltage } \\
(\mathrm{kV})\end{array}$ & $\begin{array}{l}\text { Fluoroscopic } \\
\text { time (s) }\end{array}$ & $\begin{array}{l}\text { FSC } \\
(\mathrm{cm})\end{array}$ & Zoom & $\begin{array}{c}\text { Filtration } \\
\mathrm{Cu} / \mathrm{Al} \\
(\mathrm{mm})\end{array}$ & $\begin{array}{c}\text { DAP } \\
\left(\mathrm{mGy}^{\mathrm{c}} \mathrm{cm}^{2}\right)\end{array}$ & $\begin{array}{l}\text { Fetal dose } \\
(\mathrm{mGy})\end{array}$ & $\begin{array}{l}\text { Risk of radiation } \\
\text { induced cancer } \\
(\%)\end{array}$ \\
\hline 1 & 111.00 & 130.0 & 81.3 & 48 & $0.9 / 1$ & 4464.0 & 2.94 & 0.04 \\
\hline 2 & 107.37 & 133.0 & 90.4 & 48 & $0.9 / 1$ & 4500.0 & 2.24 & 0.03 \\
\hline 3 & 117.01 & 148.0 & 79.1 & 48 & $0.9 / 1$ & 4500.0 & 3.36 & 0.04 \\
\hline 4 & 82.00 & 160.0 & 81 & 48 & $0.9 / 1$ & 7841.0 & 2.81 & 0.03 \\
\hline 5 & 94.45 & 79.0 & 92.7 & 48 & $0.9 / 1$ & 1917.0 & 0.42 & 0.01 \\
\hline 6 & 86.00 & 106.0 & 88.9 & 48 & $0.9 / 1$ & 1321.0 & 0.38 & 0.01 \\
\hline 7 & 105.67 & 254.0 & 86.9 & 48 & $0.9 / 1$ & 4278.0 & 2.38 & 0.03 \\
\hline 8 & 94.45 & 105.0 & 87.4 & 48 & $0.9 / 1$ & 2714.0 & 0.94 & 0.01 \\
\hline 9 & 102.48 & 182.0 & 80.4 & 48 & $0.9 / 1$ & 4711.0 & 2.61 & 0,03 \\
\hline 10 & 103.00 & 110.0 & 80.5 & 48 & $0.9 / 1$ & 4411.0 & 2.66 & 0.03 \\
\hline 11 & 98.00 & 169.0 & 80.7 & 48 & $0.9 / 1$ & 4114.0 & 2.42 & 0.03 \\
\hline 12 & 117.40 & 94.0 & 82.1 & 48 & $0.9 / 1$ & 3874.0 & 2.34 & 0.03 \\
\hline 13 & 111.00 & 117.0 & 85.1 & 48 & $0.9 / 1$ & 4229.0 & 2.67 & 0.03 \\
\hline 14 & 96.36 & 113.0 & 81.7 & 48 & $0.9 / 1$ & 3403.0 & 1.7 & 0.02 \\
\hline 15 & 104.61 & 161.0 & 82.1 & 48 & $0.9 / 1$ & 4030.0 & 2.57 & 0.03 \\
\hline 16 & 105.59 & 68.0 & 84.9 & 48 & $0.9 / 1$ & 2634.0 & 1.25 & 0.01 \\
\hline 17 & 80.00 & 201.0 & 81 & 48 & $0.9 / 1$ & 4532.0 & 1.5 & 0.02 \\
\hline 18 & 100.00 & 130.0 & 80.3 & 48 & $0.9 / 1$ & 2455.0 & 1.26 & 0.02 \\
\hline 19 & 101.00 & 85.0 & 82.7 & 48 & $0.9 / 1$ & 2913.0 & 1.22 & 0.01 \\
\hline 20 & 80.00 & 79.0 & 81 & 48 & $0.9 / 1$ & 2588.0 & 0.75 & 0.01 \\
\hline 21 & 107.60 & 72.0 & 84.4 & 48 & $0.9 / 1$ & 2317.0 & 1.29 & 0.02 \\
\hline 22 & 119.87 & 97.0 & 85.5 & 48 & $0.9 / 1$ & 4962.0 & 2.98 & 0.04 \\
\hline 23 & 111.00 & 104.0 & 83.1 & 48 & $0.9 / 1$ & 2751.0 & 1.4 & 0.02 \\
\hline 24 & 99.00 & 117.0 & 81 & 48 & $0.9 / 1$ & 1948.0 & 0.6 & 0.01 \\
\hline 25 & 110.60 & 124.0 & 80.3 & 48 & $0.9 / 1$ & 2457.0 & 1.23 & 0.01 \\
\hline 26 & 102.00 & 77.0 & 84.3 & 48 & $0.9 / 1$ & 1517.0 & 0.59 & 0.01 \\
\hline 27 & 80.00 & 75.0 & 81.4 & 48 & $0.9 / 1$ & 2524.2 & 0.75 & 0.01 \\
\hline 28 & 83.00 & 129.0 & 81.4 & 48 & $0.9 / 1$ & 3000.0 & 1.24 & 0.01 \\
\hline 29 & 83.00 & 107.0 & 81 & 48 & $0.9 / 1$ & 2500.0 & 0.83 & 0.01 \\
\hline 30 & 90.00 & 144.0 & 81 & 48 & $0.9 / 1$ & 1266.0 & 0.49 & 0.01 \\
\hline 31 & 80.00 & 83.0 & 85.1 & 48 & $0.9 / 1$ & 2615.0 & 0.68 & 0.01 \\
\hline 32 & 80.00 & 83.0 & 81.8 & 48 & $0.9 / 1$ & 2601.0 & 0.75 & 0.01 \\
\hline 33 & 80.00 & 155.0 & 81.8 & 48 & $0.9 / 1$ & 3190.0 & 1.13 & 0.01 \\
\hline 34 & 100.00 & 90.0 & 81 & 48 & $0.9 / 1$ & 4467.0 & 2.45 & 0.03 \\
\hline 35 & 85.00 & 83.0 & 84.7 & 48 & $0.9 / 1$ & 2475.0 & 0.81 & 0.01 \\
\hline 36 & 99.00 & 44.0 & 81 & 48 & $0.9 / 1$ & 1903.0 & 0.6 & 0.01 \\
\hline 37 & 89.00 & 129.0 & 81 & 48 & $0.9 / 1$ & 6143.0 & 2.9 & 0.03 \\
\hline 38 & 91.00 & 99.0 & 81 & 48 & $0.9 / 1$ & 3995.0 & 1.52 & 0.02 \\
\hline 39 & 82.00 & 56.0 & 84.9 & 48 & $0.9 / 1$ & 1463.0 & 0.37 & 0.01 \\
\hline 40 & 80.00 & 118.0 & 81 & 48 & $0.9 / 1$ & 2721.1 & 0.75 & 0.01 \\
\hline 41 & 83.00 & 98.0 & 81 & 48 & $0.9 / 1$ & 970.0 & 0.26 & 0.01 \\
\hline 42 & 89.00 & 50.0 & 80 & 48 & $0.9 / 1$ & 1208.0 & 0.5 & 0.01 \\
\hline
\end{tabular}

rectly into the internal iliac arteries in pregnant women, usually without needing another preshaped catheters. Our experience and the literature record slightly different morphology of the aortic bifurcation in pregnant women (almost always obtuse angle) which makes cross-over catheterisation of the internal iliac artery very easy ${ }^{17}$. Digital subtraction angiography is not used. Low dose pulse fluoroscopy should be used during the procedure to reduce $\mathrm{X}$-ray exposure ${ }^{18-20}$.

These combined procedures, Caesarean section and prophylactic occlusion of the internal iliac arteries should be performed in a hybrid operating room to avoid transporting patients from the angiography suite to the operating room which also avoids the need to reconfirm the position of the balloons and thus reduces the fluoroscopy time and fetal radiation dose ${ }^{12}$.

An experienced interventional radiologist with good knowledge of arterial anatomy can perform the procedure with low dose fluoroscopy and a short fluoroscopy time and consequent low radiation dose $\mathrm{e}^{13,21}$.

The fluoroscopy time can be shortened by occlusion of the common iliac arteries or aorta, which is an alter- 

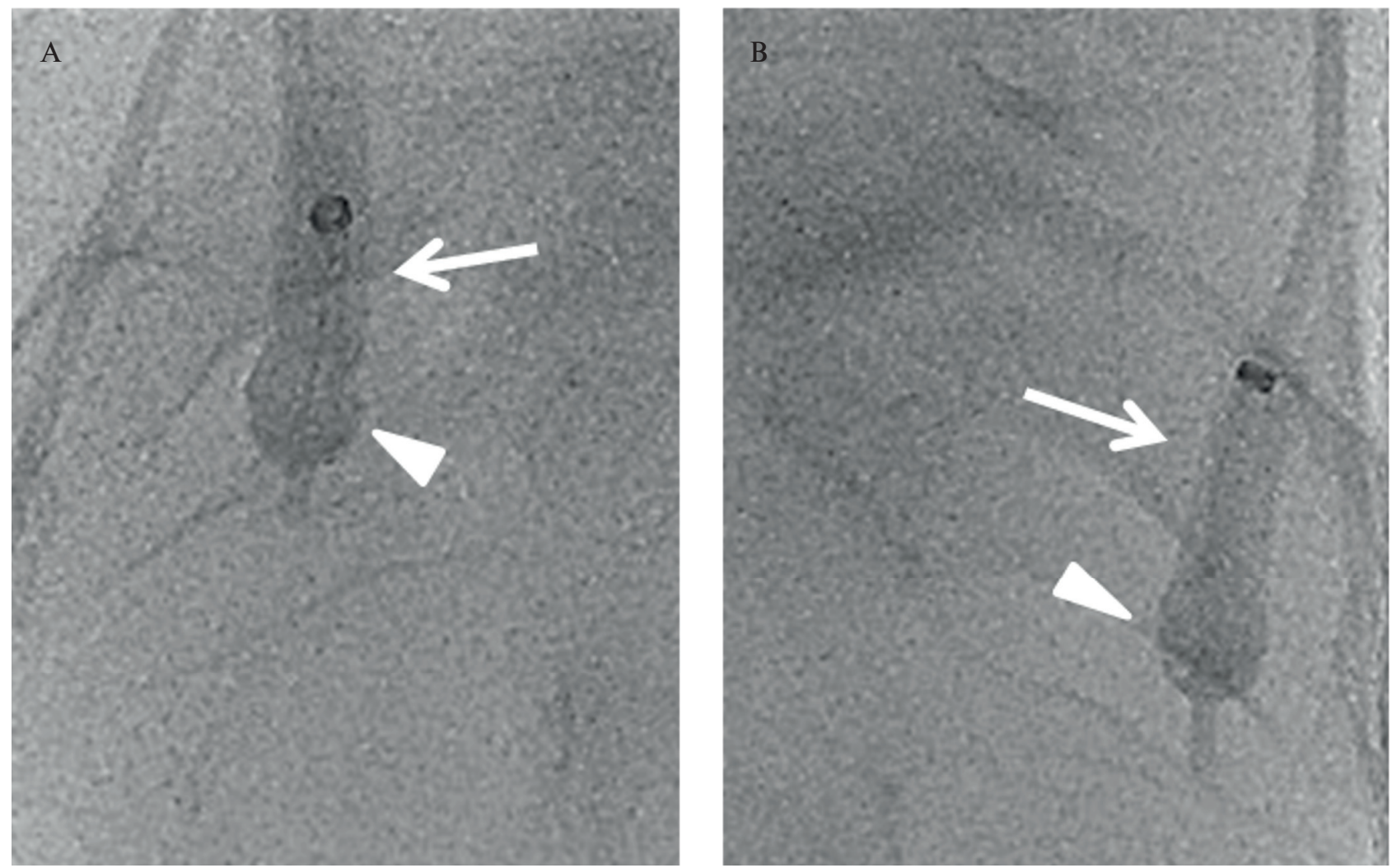

Fig. 1A,B. The record of fluoroscopy during catheter insertion. Test injection of contrast medium through the introducers to verify the occlusion of blood flow in the internal iliac arteries. Inflated Fogarty balloons (>) and stagnation of contrast medium proximally from balloons $(\rightarrow)$.

native to the most commonly used approach of prophylactic occlusion of the internal iliac arteries ${ }^{22}$. But it is necessary to consider that pregnant women have a higher risk of thrombembolic complications relating to higher artery compliance and hypercoagulation status during pregnancy ${ }^{23,24}$. Occlusion of the common iliac arteries or aorta has a higher risk of these thrombembolic complications $^{7,22,25-28}$. Furthermore, occlusion of the infrarenal aorta can be associated with aortic dissection, aortic rupture, and peripheral ischemia ${ }^{29,30}$. Additionally, supraphysiological increases in blood pressure proximal to the occluded aorta can contribute to cardiac failure ${ }^{31}$. Aortic balloon deflation and subsequent reperfusion can lead to potential cardiovascular complications ${ }^{29}$. The radiation dose can be decreased with aortic occlusion but with an associated risk of severe complications ${ }^{32}$. Placement of the occlusion balloons in the common iliac arteries can be performed even without X-ray exposure under ultrasound guidance, but with a higher risk of thromboembolic complications and risks of retrograde flow into the internal iliac arteries ${ }^{33}$. Another disadvantage is an inability to continue with uterine artery embolization in indicated cases compared with the selective catheterization of the internal iliac arteries. In our opinion, occlusion of the internal iliac arteries is easy, fast, and has a low risk of complication. In our group of patients, we had only one complication, thrombosis of common femoral artery.

In the literature we found only five articles which reported fetal radiation doses during prophylactic tempo- rary occlusion of the internal iliac arteries. Bodner et al. ${ }^{34}$ had 6 patients with placenta accreta/percreta who underwent prophylactic temporary balloon occlusion. The fluoroscopy time for placement of occlusion balloons into the anterior division of the hypogastric arteries was recorded in 3 cases. The average time was $4 \mathrm{~min} 20 \mathrm{sec}$. The average estimated fetal radiation dose was $32 \mathrm{mGy}$. Levine at al. ${ }^{35}$ reported the fetal radiation dose during prophylactic pelvic artery balloon catheterization in 5 patients with placenta accreta, the median estimated fetal radiation exposure was $61 \mathrm{mGy}$. Kai et al. ${ }^{12}$ reported the mean fetal radiation dose during prophylactic temporary occlusion of the internal iliac arteries before Caesarean section to be $21 \mathrm{mGy}$ in 3 patients with placenta praevia/ accreta. The mean fluoroscopic time was $4.86 \mathrm{~min}$. Yi et al. ${ }^{36}$ published one case of a patient with placenta increta. The fetal radiation dose was $55 \mathrm{mGy}$. The fluoroscopic time was $2 \mathrm{~min}$ and $10 \mathrm{~s}$. Semerado et al. ${ }^{13}$ reported the fetal absorbed radiation dose in 34 patients with morbidly adherent placenta during prophylactic occlusion of internal iliac arteries in Caesarean delivery. The median of fetal radiation dose was $4.65 \mathrm{mGy}$. The mean fluoroscopic time was $7.31 \mathrm{~min}$.

The mean estimated radiation dose to the fetus in our group of patients was $1.49 \mathrm{mGy}$, the median was 1.25 mGy. The mean fluoroscopy time was $1.7 \mathrm{~min}$, the median was $1.5 \mathrm{~min}$. The fetus dose in our study was lower in comparison with literature and the fluoroscopic time was shorter. The risk of radiation induced cancer in child age 
in our study was $0.01-0.04 \%$, the average risk was $0.02 \%$, and the median was $0.01 \%$.

It is important to note that, albeit small, some risk of adverse events from fluoroscopy during prophylactic occlusion of the internal iliac arteries in pregnant women with placenta abnormalities exists. To minimise this procedure should be performed by an experienced interventional radiologist in a hybrid operating room with the appropriate equipment and material. The use of low dose fluoroscopy without digital subtraction angiography is our standard practice.

\section{CONCLUSION}

We found that prophylactic temporary occlusion of the internal iliac arteries in patients with an abnormal placenta is simple and effective with minimal risk of complications and very low estimated fetal radiation dose. The technique requires appropriate endovascular device, and an experienced team working in a hybrid operating room. Although the risk of complications is small and the dose to the fetus is low, it is necessary to develop an approach which minimises the fetal radiation dose.

Acknowledgement: Supported by: IGA_LF_2020_01. Author contribution: MC: data collection, study design, literature search, manuscript writing and final approval; KH: data collection, literature search; MK: critical reading, final approval; LJ: data analysis, data interpretation; TM: data interpretation; VP: figures, critical reading.

Conflict of interest statement: The authors state that there are no conflicts of interest regarding the publication of this article.

\section{REFERENCES}

1. Hayes E, Ayida G, Crocker A. The morbidly adherent placenta: diagnosis and management options. Curr Opin Obstet Gynecol 2011;23(6):448-53.

2. Takayama T, Minakami H, Koike T, Watanabe T, Sato I. Risks associated with cesarean sections in women with placenta previa. J Obstet Gynaecol Res 1997;23(4):375-9.

3. Warshak CR, Ramos GA, Eskander R, Benirschke k, Saenz CC, Kelly TF, Moore TR, Resnik R. Effect of predelivery diagnosis in 99 consecutive cases of placenta accreta. Obstet Gynecol 2010;115(1):65-9.

4. Eller AG, Porter TF, Soisson P, Silver RM. Optimal management strategies for placenta accreta. BJOG 2009;116(5):648-54.

5. Berg CJ, Atrash HK, Koonin LM, Tucker M. Pregnancy-related mortality in the United States, 1987-1990. Obstet Gynecol 1996;88(2):161-7.

6. Committee Opinion No. 723: Guidelines for Diagnostic Imaging during Pregnancy and Lactation. Obstet Gynecol 2017;130(4):e210-6.

7. Carnevale FC, Kondo MM, de Oliveira Sousa W, Santos AB, da Motta leal Flho JM, Moreira AM, Baroni RH, Francicso RP, Zugaib M. Perioperative temporary occlusion of the internal iliac arteries as prophylaxis in cesarean section at risk of hemorrhage in placenta accreta. Cardiovasc Intervent Radiol 2011;34(4):758-64.

8. Li K, Zou Y, Sun j, Wen H. Prophylactic balloon occlusion of internal iliac arteries, common iliac arteries and infrarenal abdominal aorta in pregnancies complicated by placenta accreta: a retrospective cohort study. Eur Radiol 2018;28(12):4959-67.

9. Nicholson PJ, O'Connor O, Buckley J, Spence LD, Greene RA, Tuite DJ. Prophylactic Placement of Internal Iliac Balloons in Patients with
Abnormal Placental Implantation: Maternal and Foetal Outcomes. Cardiovasc Intervent Radiol 2018;41(10):1488-93.

10. Shahin Y, Panq CL. Endovascular interventional modalities for haemorrhage control in abnormal placental implantation deliveries: a systematic review and meta-analysis. Eur Radiol 2018;28(7):2713-26.

11. Picel AC, Wolford B, Cochran RL, Ramos GA, Roberts AC. Prophylactic Internal Iliac Artery Occlusion Balloon Placement to Reduce Operative Blood Loss in Patients with Invasive Placenta. J Vasc Interv Radiol 2018;29(2):219-24.

12. Kai K, Hamada T, Yuge A, Kiyose H, Nishida Y, Nasu K, Narahara H. Estimating the Radiation Dose to the Fetus in Prophylactic Internal Iliac Artery Balloon Occlusion: Three Cases. Case Rep Obstet Gynecol $2015 ; 170343$.

13. Semeraro V, Susac A, Morasca A, D'Antonio F, Belli AM. Foetal Radiation Dose during Prophylactic Occlusion Balloon Placement for Morbidly Adherent Placenta. Cardiovasc Intervent Radiol 2015;38(6):1487-93.

14. Wakeford R, Little MP. Childhood cancer after low-level intrauterine exposure to radiation. J Radiol Prot 2002;22(3A):A123-7.

15. Doll R, Wakeford R. Risk of childhood cancer from fetal irradiation. Br J Radiol 1997;70:130-9.

16. ICRP Publication 103. The 2007 Recommendations of the International Commission on Radiological Protection. Annals of the ICRP 2007;34 nos2-4. ISSN 0146-6453.

17. Ohlson L. Effects of the pregnant uterus on the abdominal aorta and its branches. Acta Radiol Diagn (Stockh) 1978;19(2):369-76.

18. Cho JH, Kim JY, Kang Jo, Park PE, Kim JH, Lim JA, Kim HK, Woo NS. A Study to Compare the Radiation Absorbed Dose of the C-arm Fluoroscopic Modes. Korean J Pain 2011;24(4):199-204.

19. Brown PH, Thomas RD, Silberberg PJ, Johnson LM. Optimization of a fluoroscopy to reduce radiation exposure in pediatric imaging. Pediatr Radiol 2000;30:229-35.

20. Cohen MD. Optimizing the use of pulsed fluoroscopy to reduce radiation exposure to children. J Am Coll Radiol 2008;5:205-9.

21. Andrews RT, Brown PH. Uterine arterial embolization: factors influencing patient radiation exposure. Radiology 2000;217(3):713-22.

22. Shih JC, Liu KL, Shyu MK. Temporary balloon occlusion of the common iliac artery: new approach to bleeding control during cesarean hysterectomy for placenta percreta. Am J Obstet Gynecol 2005;193(5):1756-8.

23. Broekman EA, Versteeg H, Vos LD, Dijksterhuis MG, Papatsonis DN. Temporary balloon occlusion of the internal iliac arteries to prevent massive hemorrhage during cesarean delivery among patients with placenta previa. Int J Gynaecol Obstet 2015;128(2):118-21.

24. Valentin J. Effects of in utero irradiation. Ann ICRP 2000;30(1):9-12.

25. Bishop S, Butler K, Monaghan S, Chan K, Murphy G, Edozien L. Multiple complications following the use of prophylactic internal iliac artery balloon catheterisation in a patient with placenta percreta. Int J Obstet Anesth 2011;20(1):70-3.

26. Bodner LJ, Nosher JL, Gribbin C, Siegel RL, Beale S, Scorza W. Balloon-assisted occlusion of the internal iliac arteries in patients with placenta accreta/percreta. Cardiovasc Intervent Radiol 2006;29(3):354-61.

27. Salazar GM, Petrozza JC, Walker TG. Transcatheter endovascular techniques for management of obstetrical and gynecologic emergencies. Tech Vasc Interv Radiol 2009;12(2):139-47.

28. Luo F, Wu Z, Mei J, Yue J, Yu X, Xie L. Thrombosis after aortic balloon occlusion during cesarean delivery for abnormally invasive placenta. Int J Obstet Anesth 2018;33:32-9.

29. Ribeiro MAF, Feng CYD, Nguyen ATM, Roriques VC, Bechara GEK, de Moura RR, Brenner M. The complications associated with Resuscitative Endovascular Balloon Occlusion of the Aorta (REBOA). World J Emerg Surg 2018;11:13-20.

30. Tsurukiri J, Akamine I, Sato T, Sakurai M, Okumura E, Moriya M, Yamanaka H, Ohta S. Resuscitative endovascular balloon occlusion of the aorta for uncontrolled haemorrahgic shock as an adjunct to haemostatic procedures in the acute care setting. Scand J Trauma Resusc Emerg Med 2016;24:13.

31. Russo RM, Neff LP, Lamb CM, Cannon JW, Galante JM, Clement NF, Grayson JK, Williams TK. Partial Resuscitative Endovascular Balloon Occlusion of the Aorta in swine model of hemorrhagic shock. J Am Coll Sur 2016;223(2):359-68.

32. Nieta-Calvache AJ, Salas LF, Duran EJ, Benavides SL, OrdonezDelgado CA, Rodriquez-Holguin F. Estimation of fetal radiation 
absorbed dose during the prophylactic use of aortic occlusion balloon for abnormally invasive placenta. J Matern Fetal Neonatal Med 2019;21:1-6.

33. Chen K, Zhang G, Li F, Liu J, Xie K, Zhu E, Li W, Zhang M, Gen Ch, Wang A. Application of ultrasoudn-guided balloon occluision in cesarean section in 130 cases of sinister placenta previa. J Intervent Med 2020;3:41-44.

34. Bodner LJ, Nosher JL, Gribbin C, Siegel RL, Beale S, Scorza W. Balloon-assisted occlusion of the internal iliac arteries in patients with placenta accreta/percreta. Cardiovasc Intervent Radio 2006;29(3):354-61.

35. Levine AB, Kuhlman K, Bonn J. Placenta accreta: comparison of cases managed with and without pelvic artery balloon catheters. J Matern Fetal Med 1999;8(4):173-6.

36. Yi KW, Oh M, Seo T, So KA, PAEK YC, Kim HJ. Prophylactic Hypogastric Artery Ballooning in a Patient with Complete Placenta Previa and Increta. J Korean Med Sci 2010;25(4):651-5. 